\title{
In silico Characterization and Selection of Epitope-based Peptide Vaccines Against Ebola Viruses
}

\author{
Sameer Sharma*, Sudhakar Malla \\ Department of Biotechnology, Indian Academy Degree College, Bangalore, India \\ Email address: \\ Sameer21.97@gmail.com (S. Sharma) \\ ${ }^{*}$ Corresponding author
}

\section{To cite this article:}

Sameer Sharma, Sudhakar Malla. In silico Characterization and Selection of Epitope-based Peptide Vaccines Against Ebola Viruses. International Journal of Homeopathy \& Natural Medicines. Vol. 6, No. 1, 2020, pp. 1-5. doi: 10.11648/j.ijhnm.20200601.11

Received: February 20, 2020; Accepted: March 3, 2020; Published: March 24, 2020

\begin{abstract}
Background \& Objective: To analyze the mutual B and T cell epitope related vaccine which can evoke the immune response against Ebola hemorrhagic fever. Ebola virus is pathogenic in nature which is associated with a systemic disease in man and apes. Ebola virus disease is advised to be zoonotic with random spillovers to human beings, particularly animals, and apes. These viruses can affect on immune suppression, abnormal inflammatory responses and high mortality. Methodology: In this study, membrane proteins NP, VP35, VP40, sGP, ssGP, VP30, and VP24 of Ebola virus were retrieved from the protein databases and subjected to many bioinformatics related tools to identify the antigenic B and T-cell epitopes using antigenicity analysis. The selected epitopes were subjected to molecular docking simulation along with HLA-DR to affirm their antigenicity in silico. Result \& Conclusion: The data present in our study exposed that the epitopes from NP, VP35, VP40, sGP, ssGP, VP30, and VP24 proteins might be the specific target for Ebola virus based on the best binding affinity and molecular docking score. The biochemical analysis and various characterization is also mandatory to evaluate the correlation of epitopes solely with the MHC molecules.
\end{abstract}

Keywords: Ebola Virus, Peptide Vaccine, TMHMM, BCpreds, Propred, AutoDock

\section{Introduction}

Ebola viruses are generally found in primates in West Africa and therefore the Philippines. Ebola hemorrhagic fever appears primarily in Sudan, Ivory Coast \& Uganda and also affects the many other African countries. Liability aspects for Ebola hemorrhagic fever are transit to other fields with endemic Ebola hemorrhagic fever and associate with infected people. Ebola virus extents by direct comes under contact with blood and secretions, any needle or syringes and other tools that used to operate Ebola infected peoples. Ebola viruses are negative stranded RNA dependent virus that allied to the Filoviridae family and are regional to west and central Africa [1]. Ebola viruses were classified together in newly established family named Filoviridae because of their distinctive thread like structure. The results of Ebola and Marburg hemorrhagic fever that cause agglomeration abnormalities, gastrointestinal bleeding, liver damage, and cytokine release that convey to damage vascular cells. And uncontrolled bleeding leads to blood loss and may induce hypotensive shock that leads to death in Ebola infected patients. Ebola virus disease is advised to be zoonotic with random spillovers to human beings, particularly animals, and apes. And Fruit bats are thought to be indigenous host of the Ebola virus, and although the virus has not been secluded from bats in natural conditions. Human are mainly infected through dead forest animals, and by direct contact with infected bats [2]. Moreover, the contiguous morbidity and mortality accountable to Ebola virus disease which has ambiguous effects on societies because resources are amused from programmes designed at controlling other diseases such as malaria [3-6], HIV infection [3, 4, 7, 8] tuberculosis [3, 4, 9] and human African trypanosomiasis [10]. ELISA and PCR tests are used for definitive diagnostics tests and mainly Real time PCR tests were the main technique of the laboratory response in West African Ebola virus disease outbreak. For Ebola hemorrhagic fever, there is no standard cure but only supportive therapies are available. Researchers and scientists are trying to figure out how to surmise and develop an effective vaccine against Ebola viruses with some success. 
We analyzed the investigations to find out the finest B- and T-cell epitopes from NP, VP35, VP40, sGP, ssGP, VP30, VP24 proteins of Ebola virus following antigenicity analysis and presented the binding ability of the epitopes with MHC class-I and MHC class-II by in silico molecular docking technique.

$\begin{array}{ll}\text { NP } & \text { Nucleoprotein } \\ \text { VP35 } & \text { Polymerase complex protein } \\ \text { VP40 } & \text { Matrix protein } \\ \text { sGP } & \text { Small secreted glycoprotein } \\ \text { SsGP } & \text { Super small secreted glycoprotein } \\ \text { VP30 } & \text { Minor nucleoprotein } \\ \text { VP24 } & \text { Membrane-associated protein }\end{array}$

\section{Methodology}

Prediction of antigenicity of different Ebalovirus membrane proteins: The whole amino acid sequence of each NP, VP35, VP40, sGP, ssGP, VP30, VP24 proteins of Ebalovirus were fetched from protein databases (http://www.ncbi.nlm.nih.gov/protein/) and non-identical sequences were investigated with VaxiJen (http://www.ddgpharmfac.net/vaxijen/vaxijen.html) [11]. For best accuracy, 0.5 was used as a threshold value to identify the antigenicity of each specific protein. The amino acids sequence from NP, VP35, VP40, sGP, ssGP, VP30, VP24 proteins that have antigenic score more than 0.5 were selected. For transmembrane topology analysis, each selected full length amino acid sequence was subjected using TMHMM v.2.0 (http://www.cbs.dtu.dk/services/TMHMM/) prediction server to examine exo-membrane amino acid sequences of specific protein [12].

Prediction of antigenic B-cell epitopes: Each chosen full length protein sequence was delivered to BCPreds (http://www.ailab.cs.iastate.edu/bcpreds/predict.html)

analysis in which BCPred \& AAP prediction techniques were used [13]. All selected and predicted B-cell epitopes (16mer) having a BCPreds minimal score more than 0.8 were chosen and followed by membrane topology comparing with TMHMM outcomes for exo-membrane amino acids sequences and B-cell epitope sequences (surface exposed) having the minimal value more than 0.8 for BCPreds were then examined using VaxiJen at 0.5 threshold value to analyze the antigenicity. At last, 2-3 epitopes were selected with the highest VaxiJen scores to use in prediction of T-cell epitopes.

T-cell epitopes Prediction from selected B-cell epitopes: Two screening steps were followed for prediction of T-cells epitopes from the selected B-cell epitopes. The selection criteria for first screening were 1) retrieved sequence of $\mathrm{T}$ cell epitope must bind with MHC class-I and class-II molecules and total interacting MHC molecules should be more than 15,2 ) the T-cell epitope retrieved sequence must collaborate with HLA-DRB $1 * 0101$ molecules of MHC classII, 3) T-cell epitope sequence score should be antigenic based on VaxiJen score. MHC-I and MHC-II class molecules were bind to common epitopes because of linear prediction model that utilize amino acid position coefficients through PropredI (http://www.imtech.res.in/raghava/propred1/) and Propred (http://www.imtech.res.in/raghava/propred) [14-16]. After that, total numbers of combining MHC molecules were selected \& counted. For quantitative structure activity relationship (QSAR) simulation, the half maximal inhibitory concentration (IC50) and antigenicity were calculated by MHCPred V.2 (http://www.ddgpharmfac.net/mhcpred/MHCPred/) server [17] and VaxiJen v2.0 accordingly. Highest antigenicity epitopes attached with more than 15 molecules of MHC I \& MHC II alleles and for DRB1*0101, had less than $100 \mathrm{nmol} / \mathrm{L}$ IC50 score were chosen.

QSAR screening methods were used in $2^{\text {nd }}$ screening process using T-Epitope Designer (http://www.bioinformation.net/ted/) [18] as well as MHCPred, accordingly. More than 1000 HLA allele's peptides can design using $\mathrm{T}$-epitope designer. In the $2^{\text {nd }}$ screening, the selection criteria were as follows in essence the peptide must attach more than $75 \%$ of total HLA molecules; the peptide must bind with the highest score to a) HLA-A*0204, \& HLA-B*2705, and b) DRB1*0101 \& DRB $1 * 0401$. First two criteria were analyzed using Tepitope designer and the final criteria were analyzed using MHCPred. The latest list of T-cell epitopes was done with peptide sequences and clear these mentioned selected criteria and VaxiJen and IC50 scores and physico-chemical properties of selected B-cell \& T-cell epitopes (15-mer) were identified with ProtParam computer program (http://web.expasy.org/protparam).

Molecular docking of predicted epitopes with HLA-DR: The predicted epitopes of $\mathrm{B}$ and $\mathrm{T}$-cells subjected for molecular docking with MHC I molecules (HLA-B7, and HLA-B44), and MHC class-II molecules (HLA-DRB1*0101, and HLA-DRA) [19]. Multiple sequence alignment was used for HLA super types B7, B44. (https://www.ebi.ac.uk/Tools/msa/clustalw2/). SWISSMODEL was used for 3D structure of these seven proteins (http://www.swissmodel.expasy.org) [23, 20]. PDB ID for MHC-1 super types HLA-B7 is 6avg.2.D and HLA-B44 is 4jqx.1.A. And the PDB ID of MHC class-II molecules HLADRB1*1501 and HLA-DRA were 1a6a.1.B and 3pdo.1.A, respectively. And similarity among these molecules HLA molecules was identified by multiple sequence alignment and the best molecular docking orientation was chosen based on binding free energy and hydrogen bond.

\section{Results}

Antigenicity and topology of identified proteins: The antigenicity was analyzed through auto cross covariance (ACC) transformation of protein sequences with the help of VaxiJen. According to VaxiJen score indicating the highest antigenicity of an particular amino acid sequence of NP, VP35, VP40, sGP, ssGP, VP30 and VP24 proteins of Ebolavirus was $0.4379,0.5079,0.4831,0.4813,0.5314$, 
0.5129, and 0.4779, correspondingly. And Ebolavirus proteins exhibited the VaxiJen score less than 0.5 and predicted or identified to be non-antigenic through VaxiJen. The selection criterion of a proper epitope is that it should be showed to cell outside. And another transmembrane topology of these proteins were done through TMHMM and the outcome showed that the lengths of exomembrane amino acid sequence for NP, VP35, VP40, sGP, ssGP, VP30, and VP24 proteins of Ebolavirus were 1-739, 1-340, 1-326, 1$364,1-298,1-288$, and 1-251 respectively.

Table 1. Table showing the Accession number, exo-membrane sequence \& VaxiJen score of selected proteins.

\begin{tabular}{llll}
\hline Protein & Accession number & VaxiJen score & $\begin{array}{l}\text { Exo-membrane } \\
\text { sequence }\end{array}$ \\
\hline NP & AKB09551.1 & 0.4379 & $1-739$ \\
VP35 & AKB09552.1 & 0.5079 & $1-340$ \\
VP40 & AKB09553.1 & 0.4831 & $1-326$ \\
sGP & AKB09555.1 & 0.4813 & $1-364$ \\
sSGP & AKB09554.1 & 0.5314 & $1-298$ \\
VP30 & AKB09557.1 & 0.5129 & $1-288$ \\
VP24 & AKB09558.1 & 0.4779 & $1-251$ \\
\hline
\end{tabular}

B-cell epitopes: To make a proper vaccine, a peptide must be producing B-cell and T-cell mediated immunity as well as peptide must be hydrophilic in nature [14]. BCPreds were used to analyze for B-cell epitope prediction using BCPreds and all predicted B-cell epitopes were selected on the based as already mentioned in methodology. Generally, BCPreds containing 16-mer epitopes [13] and VaxiJen minimal values

more than 0.8 and 0.5 accordingly, were listed. After the BCPreds and VaxiJen minimal values, single epitope were selected from each protein for further analysis and each listed B-cell epitope from a protein exposed $100 \%$ similarity with all amino acid sequences of that protein.
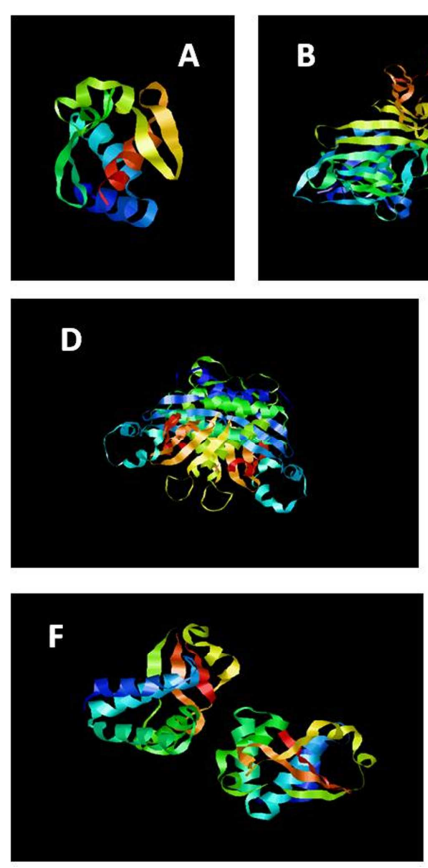

Figure 1. PDB structure of selected proteins using SWISS-MODEL a) NP, b) $V P 35$, c) $V P 40$ d) $s G P$, e) $s s G P, f) V P 30, g) V P 24$.

Table 2. B-cell epitope from full length of selected proteins.

\begin{tabular}{|c|c|c|c|c|c|}
\hline Protein name & Amino acid start position at epitope & BCPred epitope sequence & BCPred score & VaxiJen score & Exo-membrane sequence \\
\hline NP & 387 & LDDQEKKILMNFHQKK & 1.358 & 0.923 & $1-739$ \\
\hline VP35 & 83 & SQMQQTKPNPKTRNSQ & 1.294 & 0.612 & $1-340$ \\
\hline VP40 & 242 & PNKSGKKGNSADLTSP & 1.576 & 0.795 & $1-326$ \\
\hline sGP & 221 & LPQAKKDFFSSHPLRE & 0.802 & 0.865 & $1-364$ \\
\hline ssGP & 149 & YNLEIKKPDGSECLPA & 1.704 & 0.531 & $1-298$ \\
\hline VP30 & 55 & SSRENYRGEYRQSRSA & 1.997 & 0.611 & $1-288$ \\
\hline VP24 & 169 & MRTQRVKEQLSLKMLS & 1.686 & 0.877 & $1-251$ \\
\hline
\end{tabular}

T-cell epitopes selected from B-cell epitopes: T-cell epitope selection is based on B-cell epitopes. In the initial screening, MHCPred was used for identified 9-mer epitopes (EKKILMNFH, QTKPNPKTR, NKSGKKGNS, DFFSSHPLR, IKKPDGSEC, NYRGEYRQS, and QRVKEQLSL) which were all antigenic with best VaxiJen score. In the second screening level, selected peptides from the first screening were used to analyze and predict their binding capabilities to more than $1000 \mathrm{MHC}$ alleles with the help of T-epitope designer and epitopes that bound more than $75 \%$ MHC alleles were listed. For mostly used $A^{*} 0101$ and A*0102 alleles, the minimal value was set accordingly that listed peptides must bind to these HLA molecules. The final list of epitopes was made with peptide sequences that confirmed the criteria of second screening level.

Table 3. T-cell epitopes from selected B-cells epitopes sequence.

\begin{tabular}{lllll}
\hline Protein name & Predicted T cell epitope & VaxiJen score & Amino acid start position & $\begin{array}{l}\text { IC50 value of T-cell epitopes for DRBI*0101 } \\
\text { (MHCPred) (nM) }\end{array}$ \\
\hline NP & EKKILMNFH & 0.51 & 391 & 70.15 \\
VP35 & QTKPNPKTR & 0.5079 & 87 & 91.62 \\
VP40 & NKSGKKGNS & 0.5831 & 243 & 84.14 \\
SGP & DFFSSHPLR & 0.51 & 227 & 53.13 \\
SSGP & IKKPDGSEC & 0.5314 & 153 & 58.08 \\
VP30 & NYRGEYRQS & 0.5129 & 59 & 36.64 \\
VP24 & QRVKEQLSL & 0.5778 & 172 & 25.53 \\
\hline
\end{tabular}



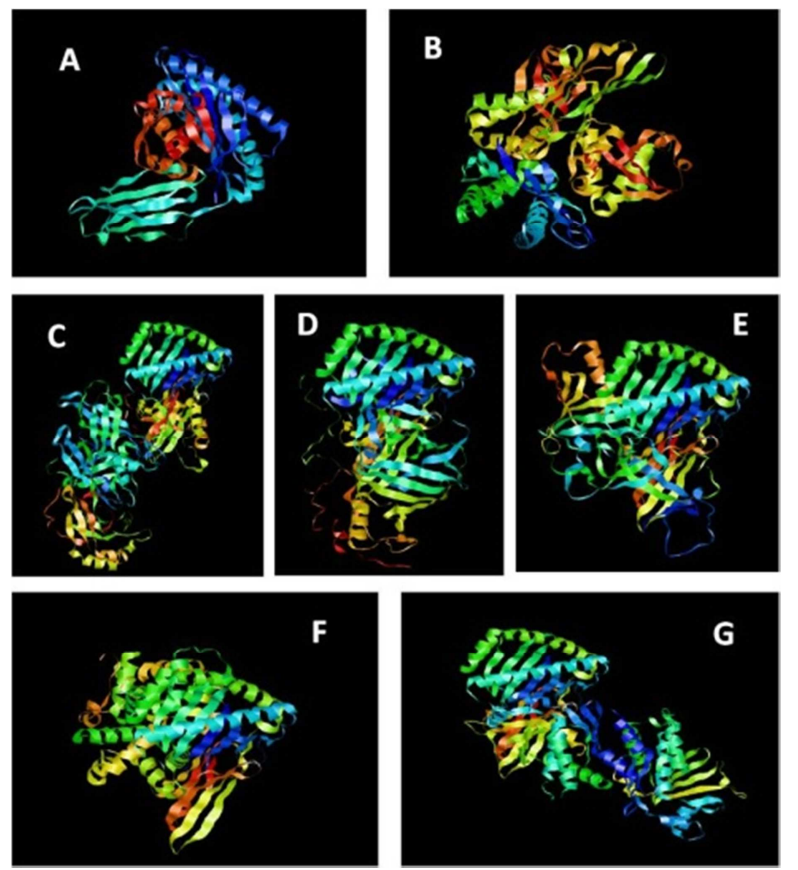

Figure 2. Molecular docking simulations of selected proteins with MHC I class molecule $B 7$ and $B 44$.
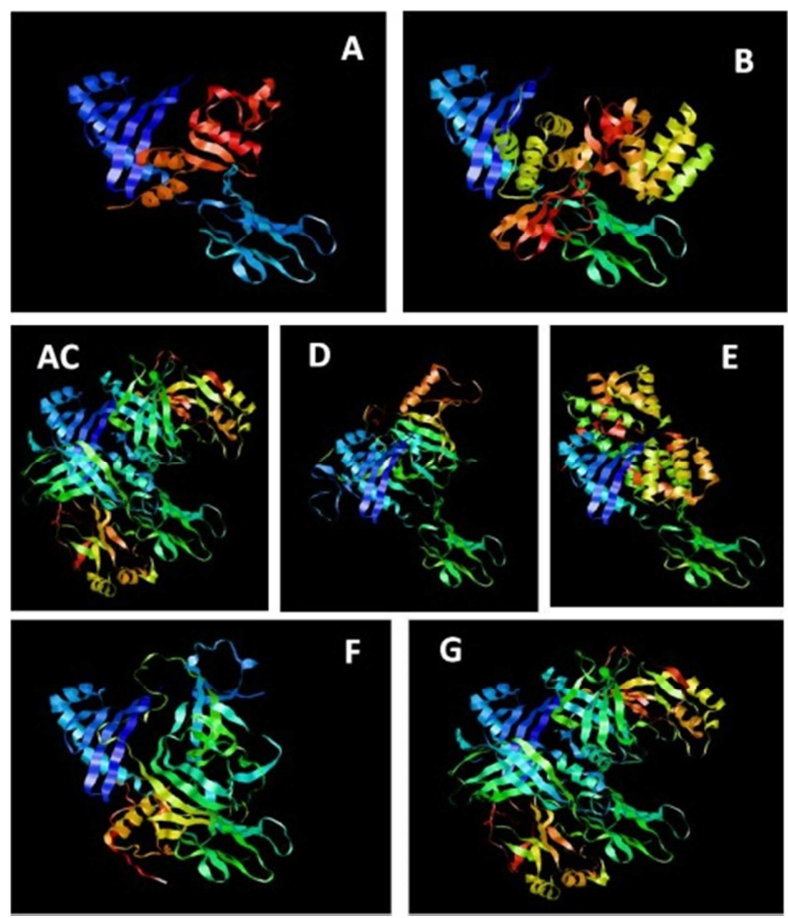

Figure 3. Molecular docking simulation of selected protein with MHC II molecules HLA-DRB1*0101, and HLA-DRB1*1501.

Molecular docking of predicted epitopes: Normally, molecular docking was used to identify the correlation between the epitopes with the MHC II molecules. Molecular docking results revealed that the HLA-DRA doesn't have collections in the peptide binding sector and serves as the solely alpha chain for HLA-DRB1 to HLA-DRB5. Molecular docking study revealed that epitope side chain attached into the grooves of HLA-DRB $1 * 0101$ and HLA-DRB $1 * 1501$ through respective hydrogen bonds. Docking outcomes also showed predicted epitope might attach with MHC II molecules in antigen presenting cells and also could trigger the $\mathrm{B}$ and T-cell epitopes. Epitope binding within the groove of the MHCII molecules is recommended as director enticement of binding affinity [21, 19]. The analysis observed that the selected proteins and listed B and T-cell epitopes bound with the groove of HLA-B7, and HLA-B44. And multiple alignment result of MHC II molecules and HLA-DRA exposed that these molecules have difference in particular sequence and as well as alignment of super types B7 and B44 showed the dissimilarity of their sequence.

\section{Discussion}

Ebola has been harmful to human health due to contagious nature since its discovery in 1976. Ebola virus is one of the most destructive forms of hemorrhagic fever which is responsible for higher number of morbidity and mortality rate. Ebola virus initially transmitted through human-tohuman contact with infected body fluids [22]. The largest outbreak of Ebolavirus disease happened in African countries from 2013-2016. Therefore, it is necessary take preventive against Ebolavirus and there is no particular vaccine for Ebolavirus. In this study, we analyzed the Ebolavirus membrane proteins which are NP, VP35, VP40, sGP, ssGP, VP30, and VP24 proteins. And the prediction of antigenicity of the non-identical sequences from membrane proteins were done with VaxiJen server [23] and TMHMM [24] prediction address used to identify exo-membrane amino acid sequence of specific protein. Antigenic B-cell epitopes predictions were done using BCPred and AAP prediction methods.

From the data, we predicted each 9-mer epitopes (EKKILMNFH, QTKPNPKTR, NKSGKKGNS, DFFSSHPLR, IKKPDGSEC, NYRGEYRQS, and QRVKEQLSL) from Ebola virus. After that, all the proteins subjected to molecular docking and the docking result with both MHC I and MHC II molecules were prominent with biologically considerable binding energy. In the current study, all the NP, VP35, VP40, sGP, ssGP, VP30, and VP24 proteins from Ebola virus showed correlation with MHC I and II molecules with prominent number of hydrogen bonds. The data exposed in this study revealed the epitopes from proteins might be specific for peptide based vaccine against Ebola virus and biochemical identification and characterization is also necessary to evaluate the interaction of epitopes solely with the MHC molecules.

\section{References}

[1] Lo TQ, Marston BJ, Dahl BA, De Cock KM, 2017. Ebola: anatomy of an epidemic. Annu Rev Med, 68: 359-70.

[2] Emanuel J, Marzi A, Feldmann H, 2018. Filoviruses: ecology, molecular biology, and evolution. Adv Virus Res, 100: 189221. 
[3] Hira S, Piot P, 2016. The counter effects of the Ebola epidemic on control and treatment of HIV/AIDS, tuberculosis, and malaria in West Africa. AIDS, 30: 2555-59.

[4] Parpia AS, Ndeffo-Mbah ML, Wenzel NS, Galvani AP, 2016. Effects of response to 2014-2015 Ebola outbreak on deaths from malaria, HIV/AIDS, and tuberculosis, west Africa. Emerg Infect Dis, 22: 433-41.

[5] Aregawi M, Smith SJ, Sillah-Kanu M, et al, 2016. Impact of the mass drug administration for malaria in response to the Ebola outbreak in Sierra Leone. Malar J, 15: 480.

[6] Plucinski MM, Guilavogui T, Sidikiba S, et al, 2015. Effect of the Ebola-virus-disease epidemic on malaria case management in Guinea, 2014: a cross-sectional survey of health facilities. Lancet Infect Dis, 15: 1017-23.

[7] Bekolo CE, Diallo A, Philips M, et al, 2017. Six-monthly appointment spacing for clinical visits as a model for retention in HIV care in Conakry-Guinea: a cohort study. BMC Infect Dis, 17: 766.

[8] Tattevin P, Baysah MK, Raguin G, et al, 2015. Retention in care for HIV-infected patients in the eye of the Ebola storm: lessons from Monrovia, Liberia. AIDS, 29: N1-2.

[9] Ansumana R, Keitell S, Roberts GM, et al, 2017. Impact of infectious disease epidemics on tuberculosis diagnostic, management, and prevention services: experiences and lessons from the 2014-2015 Ebola virus disease outbreak in west Africa. Int J Infect Dis, 56: 101-04.

[10] Camara M, Ouattara E, Duvignaud A, et al, 2017. Impact of the Ebola outbreak on Trypanosoma brucei gambiense infection medical activities in coastal Guinea, 2014-2015: a retrospective analysis from the Guinean national Human African Trypanosomiasis control program. PLoS Negl Trop Dis, 11: e0006060.

[11] Doytchinova IA, Flower DR, 2007. VaxiJen: a server for prediction of protective antigens, tumour antigens and subunit vaccines. BMC Bioinform, 8 (1): 4.

[12] Krogh A, Larsson B, Von Heijne G, Sonnhammer EL, 2001. Predicting transmembrane protein topology with a hidden Markov model: application to complete genomes. J Mol Biol, 305 (3): 567-80.
[13] EL-Manzalawy Y, Dobbs D, Honavar V, 2008. Predicting linear B-cell epitopes using string kernels. J Mol Recognit, 21 (4): 243-55.

[14] Singh H, Raghava G, 2003. ProPred1: prediction of promiscuous MHC Class-I binding sites. Bioinformatics, 19 (8): 1009-14.

[15] Singh H, Raghava G, 2001. ProPred: prediction of HLA-DR binding sites. Bioinformatics, 17 (12): 1236-37.

[16] Sturniolo T, Bono E, Ding J, Raddrizzani L, Tuereci O, Sahin $\mathrm{U}$, et al, 1999. Generation of tissue-specific and promiscuous HLA ligand databases using DNA microarrays and virtual HLA class II matrices. Nat Biotechnol, 17 (6): 555-61.

[17] Guan P, Doytchinova IA, Zygouri C, Flower DR, 2003. MHCPred: a server for quantitative prediction of peptideMHC binding. Nucleic Acids Res, 31 (13): 3621-4.

[18] Kangueane P, Sakharkar MK, 2005. T-Epitope designer: a HLA-peptide binding prediction server. Bioinformation, 1 (1): 21.

[19] Totrov M, Abagyan R, 1997. Flexible protein-ligand docking by global energy optimization in internal coordinates. Proteins, 29 (s1): 215-20.

[20] Schwede T, Kopp J, Guex N, Peitsch MC, 2003. SWISSMODEL: an automated protein homology-modeling server. Nucleic Acids Res, 31 (13): 3381-5.

[21] Patronov A, Dimitrov I, Flower DR, Doytchinova I, 2011. Peptide binding prediction for the human class II MHC allele HLA-DP2: a molecular docking approach. BMC Struct Biol, $11(1): 32$

[22] Garske T, Cori A, Ariyarajah A, et al, 2017. Heterogeneities in the case fatality ratio in the West African Ebola outbreak 2013-2016. Philos Trans R Soc Lond B Biol Sci, 372.

[23] Briand S, Bertherat E, Cox P, et al, 2014. The international Ebola emergency. N Engl J Med, 371: 1180-83.

[24] Houlihan CF, McGowan CR, Dicks S, et al, 2017. Ebola exposure, illness experience, and Ebola antibody prevalence in international responders to the west African Ebola epidemic 2014-2016: a cross-sectional study. PLoS Med, 14: e1002300. 